\title{
ГЕНЕТИЧЕСКИЙ АЛГОРИТМ ДЛЯ ВОССТАНОВЛЕНИЯ СТРУКТУРЫ ЛИНЕЙНОГО ДИНАМИЧЕСКОГО ОБЪЕКТА
}

\section{Муртузалиев Муртузали Магомедович}

д.э.н., профессор

ФГБОУ ВО «Дагестанская государственная медицинская академия»

Аннотация: Допустим, имеется некоторый объект, относящийся к классу линейных динамических систем. Сигнал $\mathrm{u}(\mathrm{t})$, поступающий на объект, является управляемым, то есть предполагается возможность постановки эксперимента. Реакция объекта $x$ на единичную ступенчатую функцию Хэвисайда, представляющая собой переходную функцию объекта, измеряется в некоторые моменты времени $t_{i}$. со случайными помехами. Данные измерений формируют обучающую выборку $\left(\mathrm{t}_{\mathrm{i}}, x\left(\mathrm{t}_{\mathrm{i}}\right)\right), \mathrm{i}=\overline{1, n}$ некоторого объема $\mathrm{n}$. Необходимо по измерениям переходной характеристики восстановить структуру и оценить параметры исследуемого объекта.Для решения такой задачи можно использоват генетический алгоритм. Рассматриваются только те объекты, поведение которых описывается линейными дифференциальными уравнениями вида

$$
\mathrm{a}_{\mathrm{m}} \frac{d^{m} x(t)}{d t^{m}}+\mathrm{a}_{\mathrm{m}-1} \cdot \frac{d^{m-1} x(t)}{d t^{m}}+\ldots+\mathrm{a}_{1} \cdot \frac{d x(t)}{d t}+\mathrm{a}_{0} \cdot \mathrm{x}(\mathrm{t})=\mathrm{b}_{0} \cdot \mathrm{u}(\mathrm{t})
$$

Так как, дифференциальные уравнения, описывающие реальные процессы, редко имеют порядок выше четвертого, ограничим область нахождения структуры объекта. Учитывая это обстоятельство, а также то, что исследования проводятся по выборочным измерениям переходной характеристики, будем искать модель в виде

$$
\alpha_{4} \cdot \frac{d^{4} x(t)}{d t^{4}}+\alpha_{3} \cdot \frac{d^{3} x(t)}{d t^{3}}+\alpha_{2} \cdot \frac{d^{2} x(t)}{d t^{2}}+\alpha_{1} \cdot \frac{d x(t)}{d t}+\alpha_{0}=\beta_{0} .
$$

Таким образом, задача построения сводится к определению шести параметров дифференциального уравнения, однако такая постановка задачи ведет к неоднозначности оптимального решения (в связи с линейной зависимостью решений). Переписав модель в виде

$$
\gamma_{4} \cdot \frac{d^{4} x(t)}{d t^{4}}+\gamma_{3} \cdot \frac{d^{3} x(t)}{d t^{3}}+\gamma_{2 .} \cdot \frac{d^{2} x(t)}{d t^{2}}+\gamma_{1} \cdot \frac{d x(t)}{d t}+\alpha_{0}=1
$$

мы не только сокращаем число настраиваемых параметров, что, в свою очередь уменьшает размерность пространства поиска, но также добиваемся единственности оптимального решения. Переведем поставленную задачу на 
язык генетического алгоритма [4]. Имеем пятимерное пространство поиска (оптимизации) действительных чисел $R^{5}$. Таким образом, каждый индивид будет представлять собой вектор этого пространства $\bar{\gamma}=\left(\gamma_{0}, \gamma_{1}, \gamma_{2}, \gamma_{3}, \gamma_{4}\right)$ фенотип, координаты которого описывают соответствующие параметры дифференциального уравнения (3). В связи с тем, что генетический алгоритм работает с генотипом, необходимо, прежде всего, определить каким образом будет осуществляться декодирования генотипа (хромосомы) в фенотип и обратно.

Ключевые слова: Алгоритм, генетика, сигнал, оптимум динамика, хромосом, фенотип, дифференциальные уравнения.

\title{
GENETIC ALGORITHM FOR RESTORING THE STRUCTURE OF A LINEAR DYNAMIC OBJECT
}

\section{Murtuzaliev M.M.}

\begin{abstract}
Let's say there is an object that belongs to the class of linear dynamical systems. The signal $u(t)$ coming to the object is controlled, that is, it is assumed that the experiment can be set up. The response of object $\mathrm{x}$ to a single step Heaviside function, which is a transient function of the object, is measured at certain points in time. with random interference. The measurement data form a training sample(ti, $\mathrm{x}(\mathrm{ti})), \mathrm{i}=(1, \mathrm{n})$ of some volume $\mathrm{n}$. It is necessary to restore the structure and evaluate the parameters of the object under study based on the measurements of the transition characteristic.To solve this problem, you can use a genetic algorithm. Only those objects whose behavior is described by linear differential equations of the form are considered

$$
\mathrm{a}_{\mathrm{m}} \frac{d^{m} x(t)}{d t^{m}}+\mathrm{a}_{\mathrm{m}-1} \cdot \frac{d^{m-1} x(t)}{d t^{m}}+\ldots+\mathrm{a}_{1} \cdot \frac{d x(t)}{d t}+\mathrm{a}_{0} \cdot \mathrm{x}(\mathrm{t})=\mathrm{b}_{0} \cdot \mathrm{u}(\mathrm{t})
$$

Since differential equations describing real processes rarely have an order higher than the fourth, we will limit the area of the object structure. Given this fact, as well as the fact that studies are conducted on sample measurements of the transition characteristic, we will look for a model in the form of

$$
\alpha_{4} \cdot \frac{d^{4} x(t)}{d t^{4}}+\alpha_{3} \cdot \frac{d^{3} x(t)}{d t^{3}}+\alpha_{2} \cdot \frac{d^{2} x(t)}{d t^{2}}+\alpha_{1} \cdot \frac{d x(t)}{d t}+\alpha_{0}=\beta_{0} .
$$


Thus, the task of building is reduced to determining six parameters of a differential equation, however, this formulation leads to an ambiguity of optimal solution (due to linear dependence of solutions). Rewriting the model as

$$
\gamma_{4} \cdot \frac{d^{4} x(t)}{d t^{4}}+\gamma_{3} \cdot \frac{d^{3} x(t)}{d t^{3}}+\gamma_{2} \cdot \frac{d^{2} x(t)}{d t^{2}}+\gamma_{1} \cdot \frac{d x(t)}{d t}+\alpha_{0}=1
$$

we not only reduce the number of configurable parameters, which in turn reduces the dimension of the search space, but also achieve the uniqueness of the optimal solution. Let's translate this problem into the language of the genetic algorithm [4]. We have a five-dimensional search (optimization) space for real numbers $\mathrm{R}^{\wedge} 5$. Thus, each individual will represent a vector of this space $\bar{\gamma}=\left(\gamma_{0}, \gamma_{1}, \gamma_{2}, \gamma_{3}, \gamma_{4}\right)-a$ phenotype whose coordinates describe the corresponding parameters of the differential equation (3). Due to the fact that the genetic algorithm works with the genotype, it is necessary, first of all, to determine how the genotype (chromosome) will be decoded to the phenotype and back.

Key words: Algorithm, genetics, signal, optimum dynamics, chromosome, phenotype, differential equations.

Генотип (хромосома) представляет собой строку символов (0и 1), которая является кодом для соответствующего фенотипа (решения). Определим длину хромосомы, а также принцип, по которому будет осуществляться переход от фенотипа к генотипу и обратно. Как было указано ранее, каждый индивид представляет собой вектор $\bar{\gamma}=\left(\gamma_{0}, \gamma_{1}, \gamma_{2}, \gamma_{3}, \gamma_{4}\right)$ пятимерного пространства. Длина хромосомы будет выбираться исходя из требований точности (число знаков после запятой) и принципов кодирования. Первоначально было выбрано следующее представление: хромосома разбивается на пять равных частей (одна часть соответствует одному определяемому параметру). В каждой части производится разбиение: первый элемент описывает знак действительного числа (0соответствует отрицательному числу,1-положительному), следующие несколько генов описывают целую частьдесятичного числа вдвоичной системе исчисления, оставшиеся идут на описание дробной части. В частности если на каждый параметр выделяется по одиннадцать генов (общая длина хромосомы составляет пятьдесятпять), при этом на целую часть приходится четыре гена, то подобное представление можно проиллюстрировать следующим образом. Первая часть (первые одиннадцать генов) хромосомы индивида [0011100011...]. Осуществим переход к фенотипу. Первый ген [00111100011...] указывает на то, что параметр отрицательный. Последующие четыре гена $[0011110001$ 1...] 
описывают целую часть числа, которая будет определяться как $\mathrm{z}=\sum_{i=1}^{4} g_{1+1} \cdot 2^{4-i}=7$. Последние шесть генов первой части хромосомы [001111000 1...] отвечают за представлениедробной части числа

$$
\mathrm{d}=\sum_{i=1}^{6} g_{i+5} \cdot 2^{6-i} / \sum_{i=1}^{6} 2^{i-1}=0(5)
$$

Конечная формула для десятичного числа имеет вид

$$
f(g)_{1}=g n_{1} \cdot\left(\sum_{i=1}^{4} g_{i+1} \cdot 2^{4-i}+\frac{\sum_{i=1}^{6} g \cdot 2^{6-i}}{\sum_{i=1}^{6} 2^{i-1}}\right)
$$

Аналогичным образом осуществляется интерпретация оставшейся части хромосомы. Однако такое представление имеет ряд недостатков. Во-первых, оно достаточно сложно реализуемо в плане обращения к генам хромосомы, представляющие не только разные параметры, но и разные части числа (знак, целую или дробную части). Во-вторых, для обеспечения высокой точности требуется значительная длина хромосомы: например, для получения результата с точностью до тысячных требуется семь генов на дробную часть для каждого параметра. В-третьих, введение отдельного гена, отвечающего за знак числа, затрудняет работу самого генетического алгоритма (подробнее об этом будет написано далее). Затем было использовано другое представление: первый ген каждой из пяти частей хромосомы выделялся на знак числа, а все оставшиеся представляли собой некое число в двоичной системе исчисления, которое делилось на некоторую константу, зависящую от требования точности и границ поиска. Первая часть (первые одиннадцать генов) хромосомы индивида [00111100011...]. Осуществим переход к фенотипу. Первый ген [00111100011...] указывает на то, что параметр отрицательный. Последующие гены [00111100011...] представляют двоичное число, переводим его в десятеричную систему исчисления

$$
\text { dec } 2 \text { bit }=\sum_{i=1}^{10} g_{i+1} \cdot 2^{10-i}=483
$$

Константа определяется из максимально возможного числа при данной длине кода (для десяти генов 1023). Таким образом, чтобы получить интервал поиска $[-5 ; 5]$, достаточно взять константу, равную двумстам. При этом будет достигнута точность приблизительно в одну тысячную. Конечная формула для десятичного числа имеет вид:

$$
f(g)=g_{1} \cdot\left(\frac{\sum_{i=1}^{10} g_{i+1} \cdot 2^{10-i}}{\operatorname{Conc}\left(\sum_{i=1}^{10} 2^{i-1}\right)}\right)
$$

Такое представление позволило избежать первого недостатка предыдущего представления (сложности), сохранив при этом третий. Выбор указанной константы позволял расширять границы поиска, уменьшая точность, 
и наоборот, однако при одновременных требованиях увеличения точности и расширения границ поиска необходимо увеличивать длину хромосомы. В качестве еще одного положительного аспекта такого представления можно отнести то, что, подобрав определенным образом константу, можно включить в возможные решения все целочисленные координаты, что, в свою очередь, при целочисленных параметрах дифференциального уравнения объекта обеспечивает наличие истинного решения в пространстве поиска. Тем не менее, отдельное кодирование знака может значительно усложнить работу генетического алгоритма: индивиды, имеющие одинаковые знаки, при скрещивании дадут индивидов с тем же знаком. Достаточно низкая вероятность мутации не сможет в должной мере скомпенсировать этот недостаток. Способом компенсации может служить процедура локального спуска, которая будет описана далее, а может быть выбрано иное представление решения, не требующее отдельного кодирования знака, и охватывающее требуемые пределы поиска.

Оченка качества решения. Оценка качества решения осуществляется по значению так называемой функции пригодности (fitness-функции) [4]. Первоначально выбирается критерий, характеризующий качество решения. Так как в работе будем искать параметры дифференциального уравнения, описывающего поведение объекта, по выборочным измерениям переходной функции, то естественно в качестве критерия использовать сумму квадратов отклонений получаемой модели от точек выборки

$$
\sum_{i=1}^{n}\left(\operatorname{fun}\left(t_{i}, \mathrm{~W}(\bar{\gamma})=\bar{\gamma}\right)-x\left(t_{i}\right)\right)^{2} .
$$

где func $\left(t_{i}, \bar{\gamma}\right)$ функция, возвращающая решение линейного дифференциального уравнения с параметрами $\bar{\gamma}$ в момент времени $t_{i}$. Понятно, что критерий использует не хромосомы, а декодированное решение.Затем находится пригодность соответствующих индивидов по формуле [4]

$$
\mathrm{f}=\mathrm{fut}\left(\operatorname{cod}(\bar{\gamma})=\frac{1}{1+W(\bar{\gamma})}\right.
$$

Очевидно, что при минимуме критерия пригодность будет максимальной (в частности, если помеха отсутствует и найденное решение является истинным, значение критерия будет равно нулю, а пригодность будет равна единице). Функция пригодности вычисляется для каждого индивида в поколении. Индивид с наибольшей функцией пригодности считается наилучшим. 
Условием останова алгоритма служит выполнение одного из двух условий: достижение заданной пригодности наилучшего индивида в поколении или установленного ограничения на число поколений.

По результатам работы можно сделать ряд выводов.

Во-первых, стоит отметить, что наблюдается расхождение параметров дифференциального уравнения, описывающего поведение объекта, и параметров найденной модели, при этом структура модели и объекта в большинстве случаев совпадает, и построенная модель достаточно хорошо описывает поведение объекта. Это объясняется спецификой самой задачи: на заданном временном интервале может быть найдены различные модели, описывающие объект. Генетический алгоритм при зафиксированных нескольких параметрах модели пытается подобрать оставшиеся таким образом, чтобы скомпенсировать имеющееся расхождение.

Во-вторых, наилучшие результаты были получены при следующих настройках генетического алгоритма: турнирная селекция (оптимальный размер турнира равен двадцати), двухточечное скрещивание. Неплохие результаты показала ранговая селекция. Пропорциональная селекция давала наихудшие результаты.

B-третьих, неравномерная мутация в большинстве случаев приводила к ухудшению результатов, однако в случае использования пропорциональной селекции наблюдалось незначительное улучшение.

В-четвертых, вид критерия качества влияет на результаты работы генетического алгоритма, причем критерий наряду с типом селекции и скрещивания может быть отнесен к параметрам алгоритма, требующим настройки. В данной работе квадратичный критерий приводил к лучшим результатам, нежели критерий, минимизирующий максимальное отклонение, в то же время последний значительно улучшал результаты при использовании пропорциональной селекции.

По сравнению с непараметрическим методом построения моделей замкнутых ЛДС, описанным в [2], генетический алгоритм позволяет получать более востребованные в практической деятельности параметрические модели и по большому счету не требует постановки эксперимента. С другой стороны он более сложен в реализации и усложняется с увеличением порядка дифференциального уравнения объекта исследования (в то время как 
непараметрический метод не зависит от структуры объекта и позволяет строить модели ЛДС любой сложности без усложнения алгоритма моделирования).

По сравнению с методом, сочетающим параметрические и непараметрические методы моделирования, описанным в [3], генетический алгоритм выигрывает в плане нахождения структуры, но несколько уступает в оценке параметров, являясь при этом более простым в реализации и удобным и универсальным в использовании.

\section{Список литературы}

1. Лагутин М.Б. Наглядная математическая статистика: учеб, пособие / М.Б. Лагутин. - М.: БИНОМ. Лаборатория знаний, 2007. - 472 с.

2. Мальцева Т.В. Построение непараметрической модели замкнутой ЛДС / Т.В. Мальцева / Молодой ученый, 2009, №10, с. 66 - 71.

3. Муртузалиев М.М., Мутуев Ч.М. Кибернетика как основа цифровизации. Учебно-метод. Пособие. Махачкала. 2019. 27с.

4. Рутковская Д. Нейронные сети, генетические алгоритмы и нечеткие системы / Д.Рутковская, М. Пилиньский, Л. Рутковский. - М.: Горячая линия - Телеком, 2007, 452 с.

5. Черноруцкий И.Г. Методы оптимизации в теории управления: учеб, пособие / И.Г. Черноруцкий. — СПб.: Питер, 2004. — 256 с. 